\title{
El gobierno contemporáneo de la exclusion social extrema en Bilbao: dispositivos integradores y procesos de autorresponsabilización*
}

\author{
Ander Mendiguren Nebreda ${ }^{1}$; Joseba García Martín²; Ivana Belén Ruiz-Estramil ${ }^{3}$
}

Recibido: 25 de octubre de 2016 / Aceptado: 17 de mayo de 2017

Resumen. El artículo propone una aproximación crítica al fenómeno de la exclusión social extrema mediante la articulación de una discusión de diversas propuestas teóricas sobre marginación, pobreza y exclusión social así como de los resultados de una investigación realizada en Centros de Asistencia Básica (CAB) dirigidos al colectivo de Personas Sin Hogar. Frente a las formas de gobierno que pretenden aproximar las situaciones de anormalidad hacia un centro normativo, el punto de partida es que la sociedad determina, por medio de la asistencia, el estatus de aquellas personas clasificadas como excluidas. Tras abordar las racionalidades que guían el programa institucional asistencial de varios $\mathrm{CAB}$ de Bilbao, analizamos, mediante técnicas de carácter cualitativo - observación participante y entrevistas-, las dinámicas de esos espacios y las prácticas que se establecen entre los profesionales y los asistidos. De este modo, hemos podido inferir la centralidad de instrumentos como el calendario, el horario y la agenda en el gobierno contemporáneo de la exclusión que, mediante la lógica del acompañamiento, pretende empoderar a los asistidos fomentando su conversión en agentes responsables de sí mismos.

Palabras clave: Personas Sin Hogar; exclusión social; centros de asistencia; dispositivo; gubernamentalidad.

[en] The contemporary government of extreme social exclusion in Bilbao: integrating gadgets and processes of self-responsibilization

\footnotetext{
Abstract: The article proposes a critical approach to the phenomenon of extreme social exclusion through the discussion of a series of theoretical proposals about marginalization, poverty and social exclusion and results in a research carried out in Basic Assistance Centers (BAC) for a group of homeless people. Against the forms of government that aim to approximate situations of abnormality to a normative center, the starting point is that society determines, through assistance, the status of those people classified as excluded. After considering the rationalities guiding institutional care programs in a number of BACs in Bilbao, we analyze, through qualitative techniques - participative observation

Todos los autores son beneficiarios del Programa Predoctoral de Formación de Personal Investigador No Doctor del Gobierno Vasco: Ivana Belén Ruiz-Estramil desde enero de 2015 y Ander Mendiguren Nebreda y Joseba García Martín desde enero de 2016. Quisiéramos, también, dejar constancia de nuestro agradecimiento a Mayra Alejandra Prado Guerrero, con quien comenzamos a problematizar la creación de redes en la extrema pobreza, y que estuvo en las discusiones iniciales de este trabajo.

1 Universidad del País Vasco/Euskal Herriko Unibertsitatea (UPV/EHU); Departamento de Sociología II. ander.mendiguren@ehu.eus

2 Universidad del País Vasco/Euskal Herriko Unibertsitatea (UPV/EHU); Departamento de Sociología II. joseba.garciam@ehu.eus

3 Universidad del País Vasco/Euskal Herriko Unibertsitatea (UPV/EHU); Departamento de Sociología II. ivanabelen.ruiz@ehu.eus
} 
and interviews - , the dynamics of these spaces and the practices being established between professionals and the assisted. This way, we have been able to infer the centrality of instruments such as the calendar, the schedule and the agenda in the contemporary government of social exclusion that, through the logics of accompaniment, aims to empower the assisted by promoting their conversion into agents responsible of themselves.

Keywords: Homeless People; social exclusion; assistance centers; device; governmentality.

Sumario: 1. Introducción al marco de la investigación. 2. Proceso etnográfico: aproximación a las zonas negativas de lo social. 3. Vidas liminales: construcción y gobierno de la pobreza. 4. Intensificación de los procesos de precarización vital y redefinición de las racionalidades institucionales. 5. Los CAB como tecnologías asistenciales contemporáneas: herramientas de reciclaje y procesos de autorresponsabilización 6. Discusión, conclusiones y futuras líneas de trabajo. 7. Bibliografía

Cómo citar: Mendiguren Nebreda, A.; García Martín, J.; Belén Ruiz-Estramil, I. (2018) “El gobierno contemporáneo de la exclusion social extrema en Bilbao: dispositivos integradores y procesos de autorresponsabilización". Cuadernos de Relaciones Laborales, 36(1), 125-145.

\section{Introducción al marco de la investigación}

La exclusión extrema es un fenómeno que en la actualidad abarca sectores alejados de las coyunturas marginales recurrentes en el imaginario colectivo (Narayan, 2002). Tal como indica el informe FOESSA, el modelo social que opera en nuestra sociedad continúa ignorando las consecuencias derivadas de aquellos "cambios que se han caracterizado fundamentalmente por la precarización de sus modos de vida" (2016: 1). Como las ondas que se crean al arrojar una piedra a un lago, la crisis económica ha golpeado no sólo a aquellos que ocupaban una posición vulnerable dentro de la estructura social, sino que ha sacudido también a aquellos que se encontraban dentro de los parámetros que constituían los procesos normativos de subjetivación contemporáneos (FOESSA, 2014) - quienes habían superado con mayor o menor fortuna las pruebas estructurales (sistema político, económico, escolar y cultural) que lo social contemporáneo les planteaba (Martuccelli, 2007) — . Derivado de este proceso, los márgenes de la pobreza y la exclusión se han ensanchando, plegándose sobre un panorama social cada vez más precarizado (Tejerina et al., 2011).

El artículo atiende a las formas de gobierno de los márgenes de lo social instituido, articulando reflexiones y experiencias surgidas a través del trabajo de campo realizado en diferentes Centros de Asistencia Básica (en adelante $\mathrm{CAB}$ ) de la ciudad de Bilbao (Bizkaia). Interesados en las formas en las que el dispositivo asistencial problematiza y gestiona la exclusión social extrema, el artículo se centra en esos espacios y tecnologías que forman parte de un amplio entramado que pretende dirigir la conducta de aquellos seres humanos que habitan los espacios liminales de la vida colectiva. Esta labor nos ha hecho retomar las consideraciones de uno de los padres de la sociología moderna, Georg Simmel (2014), y posteriormente, enmarcar nuestra mirada bajo la rúbrica de los governmentality Studies (Gordon y Miller, 1991; Miller y Rose, 2008) y las 'antropologías foucaultianas' (Biehl, 2005b; Inda, 2005; Bourgois y Schonberg, 2009). 
A pesar de constatar la intensificación de procesos de precarización vital implicados en producir devenires humanos clasificables como en situación de exclusión social (Castel, 2008), consideramos que la preeminencia de la noción de exclusión en el programa institucional es resultado de un cambio en las racionalidades desde las que se conceptualizan las desigualdades sociales (Fassin, 1999). Partiendo de esas premisas, nuestra labor se centra en comprender los discursos y prácticas que se despliegan en los $\mathrm{CAB}$, espacios que cumplen un papel decisivo dentro del Servicio Municipal de Urgencias Sociales (SMUS) desde el que se pretende satisfacer las "necesidades básicas, atendiendo a las demandas de alojamiento, alimento y vestido de forma centralizada"4. A lo largo de un trabajo de campo de dos años de duración, en los que a través de la observación participante en diferentes $\mathrm{CAB}$, y de la realización de una decena de entrevistas a técnico/as significativo/as (cuadro 1) dentro del entramado institucional municipal así como a usuarios de los mismos, hemos podido dilucidar algunas de las dinámicas de esas tecnologías asistenciales.

Frente al régimen de carácter punitivo neoliberal que atraviesa el contexto estadounidense (Wacquant, 2001; Bourgois y Schonberg, 2009), el dispositivo asistencial en el que nos hemos sumergido parece primar la intención de hacer sobrevivir y reciclar a los ciudadanos negativos para integrarlos en la vida social colectiva. En esa pulsión, los $\mathrm{CAB}$ ponen en marcha una serie de estrategias con la intención de que los sujetos asistidos recuperen las riendas de su vida, se empoderen y puedan iniciar procesos de reconstrucción del vínculo social. Entre las herramientas (Agamben, 2015) empleadas en ese proceso de recobro, el calendario, el horario y la agenda aparecen como instrumentos indispensables para tratar de ordenar la vida de seres humanos sumidos en la anomia, una lógica bajo la que reencauzar sus vidas y que aquí utilizamos como metáforas facilitadoras en el proceso de producción de ese vínculo social.

\section{Proceso etnográfico: aproximación a las zonas negativas de lo social}

Dadas las complejas características del terreno en el que hemos realizado la investigación, para situarnos y orientarnos en el campo hemos definido una situación metodológica amplia, elaborando guías de trabajo incompletas, flexibles y cambiantes (Velasco y Díaz de Rada, 2004). Por ello, desde una aproximación antropológica, hemos movilizado un conjunto de técnicas para atender a los discursos, prácticas e interacciones de los actores sociales que habitan los $\mathrm{CAB}$ (tanto trabajadores como usuarios). En concreto, han sido tres itinerarios diferentes los puestos en marcha para aproximarnos al terreno:

I) Ha sido fundamental la presencia de uno de los miembros de nuestro equipo investigador como voluntario en uno de los $\mathrm{CAB}^{5}$ de la ciudad de Bilbao, que

\footnotetext{
En su página web, el Ayuntamiento de Bilbao expone las tareas centrales de las que se encarga el dispositivo SMUS, que es puerta de entrada de todas las formas de exclusión marginal a la maquinaria institucional. Consultado el 26/02/17 en: http://www.bilbao.net/inmigracion/gestion.asp?codges=6105\&i=es.

5 Por cuestiones de anonimato y confidencialidad, no revelaremos el nombre de los entrevistados ni el de los CAB estudiados. La observación participante se ha realizado en un centro diurno de media-exigencia que es una de las ONGs pioneras en la gestión de la exclusión social extrema. Su programa se enmarca bajo el paradigma bio-psico-social de la salud mental comunitaria, la lógica del acompañamiento y la escucha.
} 
ha acudido con una regularidad de dos veces por semana a lo largo de dos años. Por medio de la observación participante ha podido adaptarse al campo, “a sus contornos y perfiles sociales que lo habitan" (Ferrándiz, 2011: 71), pasando a formar parte de la cotidianeidad del terreno. Ha podido acompañar a los usuarios por las diversas tecnologías que los gobiernan, dando pie a profundizar las relaciones empáticas con ellos y a interacciones significativas con una amplia variedad de técnicos del entramado asistencial.

II) Hemos colaborado en cuatro ocasiones en los recuentos nocturnos de personas sin hogar que se realizan de forma trimestral en la ciudad donde se desarrolla la investigación. También hemos participado en el III Estudio sobre personas en situación de exclusión residencial grave en la comunidad autónoma de Euskadi, realizado simultáneamente en 24 municipios vascos en los que se encuesta a todas aquellas personas encontradas pernoctando a la intemperie. Este estudio cuantitativo se basa en el marco conceptual desarrollado por FEANTSA (Federación Europea de Asociaciones Nacionales que trabajan con las personas sin hogar) y, concretamente, en la tipología ETHOS (European Typology of Homelessness and Housing Exclusion).

III) Hemos realizado una decena de entrevistas en profundidad, tanto a técnicos de la red institucional como a usuarios. En el caso de las entrevistas a profesionales, han sido centrales para comprender las principales racionalidades que guían las modalidades de asistencia social en nuestro contexto sociohistórico. Por otro lado, las entrevistas realizadas a los usuarios de los $\mathrm{CAB}$, nos han permitido atender al modo en el que las formas de gobierno asistencial han influido en su trayectoria vital y subjetividad. En este caso, todos los entrevistados han sido hombres, entre los cuarenta y cincuenta años ${ }^{6}$, encarnando un perfil concreto dentro de la población que se enmarca como en situación de exclusión social grave: persona sin hogar cuya situación de exclusión y marginalidad extremas se atribuye a problemas personales (drogodependencia y/o enfermedad mental).

\footnotetext{
Perfil extraído a partir de los datos recogidos por los diferentes CAB bilbaínos. El $96 \%$ de los usuarios de los $\mathrm{CAB}$ son hombres según el "III Estudio sobre personas en situación de exclusión residencial grave en la CAPV" disponible online en: https://www.irekia.euskadi.eus/uploads/attachments/8633/Dossier_de_prensa. pdf?1477638894. Aunque la edad de los usuarios de los CAB no están disponibles en las estadísticas, sin embargo, nos hemos decantado por esa franja de edad como la más significativa por tres motivos: (1) Aierdi et al. (2009) afirman que el perfil clásico es el de un hombre de mediana edad; (2) en el CAB en el que se ha realizado la etnografía el $86 \%$ de las personas atendidas supera los 35 años, un $45 \%$ las personas superan los 44 años y un $41 \%$ tiene entre 35 y 44 años; (3) nuestras experiencias e interacciones sobre el terreno han corroborado dicha información.
} 
Cuadro 1: Síntesis del trabajo de campo.

\begin{tabular}{|c|c|c|}
\hline Observación participante & 2 días/semana durante 2 años & 750 Horas. aprox. \\
\hline \multirow{4}{*}{$\begin{array}{l}\text { Recuento nocturno con } \\
\text { técnicos municipales, } \\
\text { responsables de CAB y } \\
\text { personal voluntario }\end{array}$} & Noche del 17-18 de junio de 2015 (22:00H-01:00H) & \multirow{3}{*}{$\begin{array}{c}\text { Recuento simple distribuido por } \\
\text { distritos de la ciudad y equipos } \\
\text { de trabajo. Sin interacción con } \\
\text { las personas que pernoctan en la } \\
\text { calle. }\end{array}$} \\
\hline & Noche del 16-17 de diciembre de $2015(22: 00 \mathrm{H}-01: 00 \mathrm{H})$ & \\
\hline & Noche del 15-16 de junio de 2016 (22:00H-01:00H) & \\
\hline & $\begin{array}{l}\text { III Estudio sobre personas en situación de exclusión residencial } \\
\text { grave en la CAPV. Noche del } 26-27 \text { de octubre } 2016(21: 00 \mathrm{H}- \\
01: 00 \mathrm{H})\end{array}$ & $\begin{array}{l}\text { Realización de un cuestionario a } \\
\text { todas aquellas encontradas } \\
\text { pernoctando en la calle por } \\
\text { distritos y equipos de trabajo. }\end{array}$ \\
\hline \multirow{3}{*}{$\begin{array}{l}\text { Entrevistas en profundidad a } \\
\text { informantes clave }\end{array}$} & Técnicos municipales & TEC1; TEC2; TEC 3 . \\
\hline & Personal responsable de los $\mathrm{CAB}$ & $\begin{array}{c}\text { CAB1; CAB2; CAB3; CAB4; } \\
\text { CAB5. }\end{array}$ \\
\hline & Usuarios de $\mathrm{CAB}$ & USU1; USU2. \\
\hline Notas de campo & \multicolumn{2}{|c|}{$\begin{array}{l}\text { Puesta en común y discusión de las notas de campo y el material audiovisual producido por cada uno de } \\
\text { los investigadores en cada uno de las participaciones en el terreno }\end{array}$} \\
\hline
\end{tabular}

Fuente: Elaboración propia.

Estas incursiones en el campo han estado mediadas por lecturas y teorías que han aportado recursos y contribuido a perfilar nuestro enfoque, y a su vez, hemos ido reelaborando "los conceptos adoptados para hacerlos corresponder con las sutilezas de los hechos observados" (Augé y Colleyn, 2005: 11). Todo ello ha activado nuestra "imaginación etnográfica" (Willis, 2000), edificando marcos explicativos "rozándonos, cuando no rasguñándonos o directamente chocándonos con las relaciones y procesos sociales" (Ferrándiz, 2011: 50).

Nos distanciamos de aproximaciones positivistas interesadas en la producción de conocimiento de tipo estadístico que objetiva y hace emerger el fenómeno de la 'exclusión social' como la "Encuesta a personas sin hogar" —realizada por el Instituto Vasco de Estadística - o el "Estudio sobre personas en situación de exclusión residencial grave" - en el que participamos - Aunque es evidente que la cuantificación es necesaria para el rendimiento óptimo del entramado asistencial ${ }^{7}$, consideramos que la información estadística, al contar y categorizar, hace emerger nuevas clases de colectivos humanos y objetiva sus formas de estar en el mundo (Hacking, 1990).

En cuanto a la localización empírica de nuestra investigación, debemos resaltar que se trata de un campo muy fértil para analizar las formas de gobierno asistencial contemporáneas. Tal como afirman Aierdi et al. (2009), los servicios dirigidos a las personas sin hogar en la Comunidad Autónoma del País Vasco se concentran en Bilbao (una de las ciudades que proporcionalmente más tiene del Estado) conformando

\footnotetext{
Cuestión en la que insistieron mucho los técnicos que guiaban los recuentos y el estudio sobre personas en situación de exclusión residencial grave. A través de las cifras recabadas las instituciones actuarían con mayor o menor presupuesto, optimizando el funcionamiento del aparato asistencial adecuándolo a las necesidades que la 'realidad' evidenciara a través de los informes de corte cuantitativo.
} 
una gran organización que ofrece una amplia cobertura. Se trata de un entramado que ha sido posible asentar, en parte, a través de la externalización de las funciones de asistencia sobre organizaciones no gubernamentales laicas y religiosas que cuentan con una trayectoria más amplia.

El ayuntamiento tiene la obligación de atender las necesidades de alimentación de sus ciudadanos (...). No es que quiera atenderlo, tiene que. Los comedores que estamos, son comedores que surgen hace muchos años desde ámbitos religioso (...). Entonces, comedores que ya existen el ayuntamiento los convenía en vez de crear nuevos, pero la competencia es suya (CAB1).

Como garantes del bienestar de los habitantes de la ciudad, el área de acción social de la Diputación Foral de Bizkaia y el Ayuntamiento se comprometen a financiar ese entramado de servicios sociales (Ararteko, 2006). La cobertura de la organización establece en una suerte de red que cubre las diferentes conexiones entre la asistencia y las distintas necesidades de los colectivos, intentando completar el objetivo de atender sus necesidades específicas. Entre estas tecnologías hay que destacar: 1) los acogimientos de urgencia que ofrecen una solución residencial de carácter temporal, una obligación legal para los municipios que tienen una población superior a 20.000 habitantes; 2) los comedores sociales; 3) centros de baja y media exigencia, que tratan de ofrecer espacios para 'no estar en la calle'; y 4) un equipo de calle que asiste, sobre todo, a aquellos que 'duermen a la intemperie'.

Todos estos servicios son gestores del espacio negativo de la vida colectiva en la ciudad de Bilbao, la precariedad económica y vital extrema que se contrapone a la positividad normativa. En este sentido, nuestro interés por adentrarnos en esos espacios - los $\mathrm{CAB}$ - , en comprender sus lógicas y las formas de vida que los habitan, se encuadra en una propuesta más ambiciosa que se interroga sobre la posibilidad de elaborar marcos interpretativos que comprendan formas de vida precarizadas y expulsadas de las dinámicas sociales definidas como normales y deseables. Se trata de un acercamiento a aquello que Zygmunt Bauman (2005) define en términos de residuos humanos, que, a pesar de ser identificados en los albores de las ciencias sociales, han sido dotados de mayor centralidad y materialidad por las corrientes contemporáneas críticas (Bourdieu et al., 1999; Butler, 2002). Estas nuevas formas de hacer sociología enfatizan la necesidad de acercarse a los pobladores del afuera discursivo, seres aparentemente "monstruosos", para cuestionar los "marcos de reconocibilidad' instituidos en un determinado periodo sociohistórico (Butler, 2010).

Tal como afirma Foucault (1978), la ventaja de adentrarse en esas realidades anómicas, de atender a esos espacios negativos de la vida social, es que permite comprender mejor el sistema de racionalidades de la vida social normativa. En este sentido, situarse en el margen implica desvelar las lógicas que configuran los límites de ese 'exterior constitutivo' del campo de los sujetos (Butler, 2002). Frente a los acercamientos que tratan de analizar los fenómenos sociales explicando su contenido positivo, consideramos que es en los restos de la vida social donde emerge "lo que una sociedad reconoce y admite positivamente y lo que por esa misma sociedad, por esa misma cultura, es excluido y rechazado" (Foucault, 1978: 75). Nos interesa atender al modo en el que, mediante prohibiciones, imposibilidades y exclusiones, se edifican las fronteras divisorias entre los definidos como normales y las formas de vida humanas "pero expulsadas del discurso que las hace sociales (...), incluidas 
en él sólo en los parágrafos que administran sus bordes" (Gatti, 2014: 203-204). La producción de la normalidad precisa del juego de la différance, que se sostiene por medio de la exclusión "a través de la construcción discursiva de un afuera constitutivo y la producción de sujetos abyectos y marginados, aparentemente al margen del campo de lo simbólico" (Hall, 2003: 35).

\section{Vidas liminales: construcción y gobierno de la pobreza}

Para abordar ese espacio de la vida social poblado por seres categorizados como Personas Sin Hogar (PSH), a lo largo del trabajo de campo nos hemos ido apropiando de una serie de herramientas de focalización con las que perfilar el terreno y definir los caminos a tomar. Partimos de una postura crítica ante miradas que naturalizan la situación de formas de vida precarizadas como resultado de déficits del individuo y/o la comunidad. Estas racionalidades - desde las que se programan políticas sociales integradoras que tratan de ayudar a sujetos y colectivos a superar sus incapacidades - están presentes en los discursos de organismos internacionales y subyacen a la configuración del IDH (Campana, 2014). Según nuestro criterio, se trata de discursos acríticos que obvian las dinámicas sociohistóricas implicadas en la producción de vidas precarizadas.

Para relativizar la condición de PSH, recurrimos, en primera instancia, al análisis de la condición del pobre ${ }^{8}$ elaborada por Simmel, quien aporta claves teóricas centrales para entender dicho fenómeno como una producción social, histórica y relacional (Paugam, 2007). En su ensayo El Pobre (2014), Simmel propone argumentos de gran profundidad analítica sobre los que hemos fundamentado nuestra aproximación teórica:

El pobre, como categoría sociológica, no es el que sufre determinadas deficiencias y privaciones, sino el que percibe socorros o debiera recibirlos según las normas sociales. Así considerada, la pobreza no puede definirse en sí misma y por sí misma como un estado cuantitativamente determinado, sino sólo en función de la reacción social que nace de una situación específica (2014: 79).

Se trata de un planteamiento que desesencializa plenamente el estatus del pobre, mostrando que no es una condición medible, sino que se configura en función de su interrelación con la sociedad, siendo "la estructura general del contexto social la que decide la cuestión del lugar que ocupan" (ibídem: 23). Recibir —o estar en situación de recibir - asistencia es la marca que define a determinados seres humanos como pertenecientes al estrato social más desvalorizado, sufriendo una particular exclusión por parte de la colectividad que los atiende: forman parte de la totalidad, pero en situación marginal (ibídem).

Proponemos una relectura foucaultiana para analizar las formas en las que se gestionan aquellos seres que encarnan la categoría de PSH. Se trata de una reorientación teórica que nos insta a defender, apoyándonos en los postulados de la filosofía

Alexis de Tocqueville, en su Memoria sobre el pauperismo (2003), aportó claves analíticas cercanas a las de Simmel. Elaboró una crítica política a la asistencia legal permanente como productora de una categoría, la de los asistidos, individuos perezosos y sin moral. 
continental pos-estructuralista, el carácter contingente, histórico e inventado de la existencia humana (Gordon y Miller, 1991). Desde esta mirada, en vez de atender a cifras económicas y sociodemográficas, tratamos de comprender cómo las modalidades de gestión desplegadas en un periodo sociohistórico sujetan determinadas formas de vida — en este caso, precarizadas - estructurando sus posibles campos de acción (Foucault, 2001).

Aunque somos conscientes de que los devenires humanos precarizados son resultado de dinámicas mucho más amplias que expulsan esas vidas a los espacios negativos de la vida social, consideramos que el entramado asistencial en el que nos hemos sumergido influye en la existencia de los asistidos. Nuestra pretensión, apoyándonos en el aparato conceptual de los estudios de gubernamentalidad anglofoucaultianos y las 'antropologías foucaultianas' (Inda, 2005), es descifrar algunas de las dinámicas desplegadas en ese dispositivo, definido como:

Un ensamblado de formas prácticas de conocimiento, con modos de percepción, prácticas de cálculo, vocabularios, tipos de autoridad, formas de juicio, formas arquitectónicas, capacidades humanas, objetos y aparatos no-humanos, técnicas de inscripción y un lago etcétera, todos ellos atravesados y seccionados por las aspiraciones de alcanzar ciertos resultados en términos de la conducta de los gobernados (Rose, 1999: 52).

Abordaremos dicho ensamblaje a partir del concepto de gubernamentalidad, definido como la fusión procesual -contingente, dinámica e inestable- de racionalidades políticas y tecnologías de gobierno (Musseta, 2009). Las racionalidades son programas explícitos en los que se conceptualiza el ejercicio del poder para resolver fenómenos que han sido conceptualizados como problemas que deben ser gobernados. Programas donde el saber experto, debido a su capacidad de producir discursos de verdad, tiene una centralidad especial (Miller y Rose, 2008). Por otro lado, las tecnologías son "«inocentes» mecanismos, ritualizados, banalizados, casi naturalizados por su aplicación habitual en la práctica cotidiana, a través de los cuales las autoridades buscan «instanciar» las prácticas de gobierno" (De Marinis, 1999: 89). Además, este planteamiento posibilita vincular el análisis de las formas de gobierno con la hermenéutica del sujeto (ibídem); y en este sentido, hay que atender a las 'líneas de subjetivación' del dispositivo (Deleuze, 1990), el modo en el que su carácter performativo (Butler, 2002) hace mella en la carne y mente humanas produciendo sujetos.

Analizaremos los $\mathrm{CAB}$ como tecnologías de gestión atravesadas por discursos que identifican atributos negativos en determinados seres humanos para definirlos como administrables y, en consecuencia, dirigir su conducta. Tal como explica Gatti:

Quien habita ahí, en el borde de la buena sociedad, en la esquinita, es el pobre, el carenciado, el excluido, también el extranjero... Está en el borde, pero no es un sujeto exterior a la sociedad: es el último extremo de la vida social, pero es un con parte, y merece por eso que se trabaje sobre él (moralmente, educativamente, sanitariamente, policialmente...) para integrarlo, para acercarlo al núcleo -ese lleno de ciudadanos normales- del que, al menos teóricamente, es potencialmente miembro (2014: 201). 
En función de las racionalidades imperantes en un determinado contexto sociohistórico, esos seres humanos negativos estarán sujetados a diferentes marcos que justifican diferentes formas de intervención. Podrán ser definidas como vidas vulnerables dignas de asistencia social, o por el contrario, ser enmarcadas como delincuentes que deben ser gobernados mediante políticas de tolerancia cero. En este sentido, la comparación con otras formas de gestión en el tiempo y en el espacio han fundamentado nuestra capacidad analítica. Para dicha labor, han sido indispensables una serie de lecturas realizadas: Vigilar y Castigar (Foucault, 2012) nos ha permitido constatar una mutación en las formas de gestión contemporáneas; la labor etnográfica de João Biehl (2005a; 2005b; 2007), a pesar de localizarse en un contexto muy diferente, ha sido una gran fuente de inspiración tanto en el desarrollo del trabajo de campo como a nivel conceptual; Righteus dopefiend (2009) de Bourgois y Schonberg, así como otros textos de Philippe Bourgois (2010) y Loïc Wacquant $(2001 ; 2012)$ nos han ofrecido un marco de comparación con la gestión predominante en EEUU; los trabajos etnográficos de Veena Das y Deborah Poole (2008), en donde los márgenes de la vida social, a menudo vistos como espacios de desorden, son conceptualizados como supuestos necesarios del estado; y finalmente, las aportaciones de Didier Fassin, con su idea del gobierno de la vida y del sufrimiento (1999), la razón moral esgrimida en las acciones asistenciales (2005), así como la biopolítica operante sobre los entornos de exclusión social (1996).

\section{Intensificación de los procesos de precarización vital y redefinición de las racionalidades institucionales}

Muchos analistas constatan que la realidad contemporánea, debido a un conjunto de cambios sociales, económicos, culturales y políticos, como los procesos de individualización y mundialización (Pérez-Agote, 1996), se caracteriza por el menoscabo de los centros ordenadores de la acción de la modernidad que dotaban de una aparente lógica unitaria a los sujetos (Lewkowicz, 2004; Dubet, 2010; Tejerina et al., 2011). La paulatina erosión de las lógicas integradoras características del periodo fordista - fundamentalmente, el empleo y el Estado de bienestar-, al compás de los procesos de desregulación, flexibilización y liberalización, han generalizado un clima cargado de incertidumbre y desprotección (Bauman, 2005).

Estos procesos que atraviesan las sociedades contemporáneas posfordistas afectan, cada vez más, a "grupos de personas (...) que en condiciones normales están muy lejos del estereotipo del vagabundo, transeúnte, alberguista" (Cabrera, 1998: 42). La reproducción de esas lógicas que desestabiliza incluso a los estables (Castel, 1997), ha dado lugar a la multiplicación y diversificación de formas de vida precarizadas, que ahora proceden de categorías sociales heterogéneas (Cabrera, 1998; Paugam, 2007). Por ello, siguiendo la clasificación de Paugam (2007), las múltiples experiencias de la pobreza descalificadora se asimilan a las de la marginalidad ${ }^{9}$. En el caso del concepto de PSH, definido como el estadio extremo de la exclusión social y empleado por la mayoría de investigaciones como una alternativa a los conceptos

No pretendemos negar la porosidad y las posibles conexiones entre esos tipos ideales -no son compartimentos estancos-, pero consideramos necesario destacar que el modo en el que la sociedad las gestiona, así como las representaciones que las determinan y las vivencias de quienes las padecen son diferentes. 
más vinculados a la condición indigna de la pobreza (vagabundo, indigente o mendigo) y a los que enfatizan excesivamente la cuestión residencial (sin techo), acoge a un colectivo que está compuesto por perfiles heterogéneos:

El clásico, compuesto por un hombre de mediana edad, con problemas de toxicomanías y alcoholismo y un alto grado de marginalización; aquellos perfiles emergentes que engloban nuevas situaciones y colectivos, caracterizados por personas tanto jóvenes como de mediana edad y menos relacionadas con consumos de drogas; y sobre todo, el más destacable por su cuantía y características, el de las personas inmigrantes, en su mayoría jóvenes provenientes de los países del Magreb. Un cuarto perfil a destacar, transversal a los anteriores, ya que incluye personas de los tres grupos, sería el de personas con enfermedad mental o con patología dual, colectivo éste que va en aumento con el paso del tiempo (Aierdi et al., 2009: 55).

En el terreno en el que nos hemos sumergido conviven múltiples formas de vida precarizadas. Tal como afirma Robert Castel, situar todas esas vidas bajo la misma conceptualización de la exclusión implica:

Pasar por alto el hecho de que no tienen el mismo pasado ni el mismo presente ni el mismo porvenir, y que sus trayectorias son totalmente diferentes. Es hacer como si vivieran en un espacio fuera-de-lo-social (2008: 63).

Por ejemplo, podría aplicarse la clásica distinción entre «nuevos y viejos pobres» (Gueslin, 1998), dos conceptos que nos sirven para distinguir entre las problemáticas derivadas por la incidencia de la crisis económica - trayectorias cortas debido a cuestiones coyunturales - y aquellas otras trayectorias - que pueden ser convulsas o no- de más larga duración por motivos que se definen en términos individuales - drogodependencias, cárcel y problemas de salud mental—. A su vez, también habría que atender a las experiencias de aquellos determinados por la experiencia migratoria, sujetos que son ejemplos paradigmáticos de interseccionalidad debido a su condición de extranjeros.

Yo trabajada de repartidor de barriles de cerveza. Al cabrón este [su antiguo jefe], le han pillado robando barriles allí en la Heineken. Son autónomos los que hacen el reparto (...). Cuando mi amigo trabajaba con él le había dado algo en la espalda por los barriles y me dice ¿quieres trabajar? Para seis meses antes de marcharme que me quedan, trabajo. Y me dice el jefe, mira, en tres años me jubilo, si quieres te dejo las rutas, tú me pagas el camión (...). Pero cuando ha cambiado a otra empresa, lo han buscado y le han pillado (...) y al final le han echado. Y me ha tocado a mí... iban todas las cosas perfectas, casi perfectas y se ha jodido la historia (USU1).

A pesar de coincidir con las constataciones relativas a la intensificación de procesos de precarización vital, nuestro interés se centra en cuestionar las racionalidades que atraviesan el entramado asistencial contemporáneo. Siguiendo a Fassin (1999) y Castel (2008), consideramos que la conceptualización relativa a la exclusión social ${ }^{10}$,

${ }^{10}$ La propia noción de 'exclusión' ha sido objeto de resignificaciones. Tal como afirma Paugam, en los 'Treinta 
que cristalizó en el programa institucional europeo a partir de la década de 1980 (Silver, 1994), responde a una novedosa redefinición de las desigualdades sociales que condiciona las políticas que pretenden luchar contra ellas. Esther Raya (2005) afirma que existe una falta de consenso entre las múltiples definiciones de la noción ${ }^{11}$, una indeterminación que se acentúa debido a su doble uso, como término de política social y como concepto sociológico (Herzog, 2011).

En general, la mayoría de conceptualizaciones de la exclusión subraya la centralidad de la falta de integración, estado que tiende a describirse como resultado de un "aflojamiento del lazo social que habría marcado la ruptura de los individuos respecto de sus inserciones sociales para dejarlos frente a sí mismos y a su inutilidad" (Castel, 2008: 63). Según Fassin, se trata de una renovación discursiva que implica una representación del espacio social en términos de estructura horizontal: "un interior compuesto por los integrados y un exterior que reúne a los excluidos" (1999: 34).

En la configuración de la nueva cuestión social parece que se continúa esbozando un cuadro de la vida colectiva en el que se reproduce el esquema clásico en el que existe un ficcional adentro de la sociedad, poblado por seres pertenecientes a la misma, mientras que, oponiéndose a este, se ubican personas que habitan un espacio marginal que debe ser gestionado. Se trata, advierte Fassin, de una representación de la desigualdad que no produce crítica, sino adaptación:

Se puede hablar de arreglos internos que implican mínimas correcciones, lo cual significa que actualmente se considera prácticamente imposible luchar contra las desigualdades; sólo se lucha contra sus consecuencias más visibles (1999: 36)

Los programas asistenciales diseñados en base a esos discursos dejan de centrarse en el mundo del trabajo y la redistribución de la riqueza, para atender a los excluidos bajo el imperativo de la integración a través de medidas que pretenden aliviar su situación, sin modificarla (Castel, 2008). Se ha producido un desplazamiento de los problemas sociales a la periferia de nuestras sociedades; es decir, "en vez de actuar sobre el núcleo de los problemas, donde se producen, estos son trasladados a los márgenes, separándolos de su contexto de producción" (Herzog, 2011: 611).

La idea no es que venga sólo a comer a Bilbao, la idea es que siempre habría que trabajar con ellos, porque muchos de los que comen en el comedor, está durmiendo en albergues, entonces hay otros compañeros que están en albergues y que están en contacto con ellos, los educadores que están en calle y luego los comedores, y desde ahí intentamos coordinarnos. Y el objetivo es que pueda salir, que no se estabilicen en esas situaciones que la gente vaya incorporándose" (CAB1).

Estas racionalidades tienden a reproducir la clásica dicotomía inclusión/exclusión, centrando su atención en los excluidos e instan a pensar la asistencia de las formas de vida precarizadas obviando el carácter político, social e histórico de su des-

Gloriosos', "no designaba (...) el fenómeno de deterioro del mercado de trabajo y de debilitación de los lazos sociales, sino más bien la supervivencia visible y vergonzosa de una población mantenida al margen del progreso económico" (2007: 147).

11 Desde las que otorgan mayor responsabilidad al individuo (Martin, 2011) hasta las que profundizan más en las dinámicas estructurales (Subirats, 2005). 
tino, tratándolas como víctimas vulnerables con déficits que las convierte en sujetos dignos de asistencia social bajo el imperativo de la integración (Fassin, 1996; 1999). La conceptualización de la exclusión, a diferencia del reduccionismo economicista del término pobreza, enfatiza las carencias de los sujetos en diversas dimensiones que les distancian del ciudadano ordinario (económico, laboral, formativo, sociosanitario, residencial, relacional, político y espacial). Así, refuerza una representación concreta de ciudadano positivo y las fronteras que expulsan seres humanos al espacio negativo de la vida colectiva.

A veces es muy difícil ¿no? una persona que está en la calle cómo coño vamos a hacer que aporte algo socialmente, bueno, ya está haciendo una aportación social que no reconocemos, que es... hacernos ver que hay gente que está peor que nosotros. Y esta es una aportación muy importante para... La gente mientras sepa que hay alguien que..., y lo vea, que alguien está mucho peor, se consuela de sus propios problemas. Es algo muy reactivo y muy feo pero funciona y es muy efectivo (TEC3).

De todas formas, aunque los discursos de la exclusión social predominan en la gubernamentalidad asistencial contemporánea occidental, es necesario distinguir entre la existencia de dos modelos de gestión. En el contexto anglosajón, especialmente en EE.UU., la noción de undersclass, que enfatiza la falta de voluntad y la responsabilidad individual de los 'inadaptados', ha predominado frente a la conceptualización más europea de la exclusión (Herzog, 2011). Por ello, se ha configurado una gubernamentalidad punitiva neoliberal (Bourgois, 2010) dirigida a la destrucción de residuos mediante políticas de tolerancia cero (Wacquant, 2001; Bauman, 2005).

En nuestro contexto, a pesar del progresivo desmantelamiento del Estado benefactor y la primacía de políticas neoliberales, sigue prevaleciendo un modelo en el que continúa siendo importante la intención de reciclar, "de «rehabilitar», «reformar», «reeducar» y devolver al rebaño la oveja descarriada" (Bauman, 2005: 113). Desde un enfoque más progresista que se apoya en los discursos de la exclusión, se despliega un tratamiento paternalista mediante el desarrollo de tecnologías de carácter asistencial. Dominan formas de gobierno gestadas en la compasión, en lo humano, que configuran formas benévolas para el tratamiento de la otredad "cuyo objetivo será siempre sacar a estos individuos del espacio de la exclusión, procurando su integración en la sociedad" (Irazuzta y Martínez, 2014: 29).

Se trata de una dinámica que, en base al discurso oficial de la asistencia social, se piensa desde las erosionadas ruinas de la modernidad: "la idea de ciudadanía, la integración en ella, sus dictados y sus prohibiciones, sus armonías y desajustes" (Gatti, 2014: 209). Es un paradigma que en el nivel empírico muestra evidencias claras de debilidad, porque "las situaciones precarias, los restos, los residuos, son un mecanismo asociado al propio funcionamiento social" (Tejerina et al., 2011: 22). Estamos ante una multiplicación de seres humanos en situación de despojo, de modo que "el volumen de residuos humanos crece más deprisa que la capacidad de gestionarlos" (Bauman, 2005: 94). A pesar de que los contenedores de la modernidad desbordan, incapaces de cumplir sus funciones explícitas de reciclaje, las racionalidades hegemónicas del entramado asistencial continúan legitimando la intención de aproximar las anormalidades hacia el centro normativo. 


\section{Los CAB como tecnologías asistenciales contemporáneas: herramientas de reciclaje y procesos de autorresponsabilización}

Hemos podido ir desentrañando el complejo entramado asistencial, un dispositivo en el que encontramos múltiples medidas que son resultado de una plurificación de tecnologías sociales (Rose, 1998). Dicha diversificación, característica de la gubernamentalidad liberal avanzada, introduce cierta flexibilidad en la economía de las protecciones para ajustarse a la especificidad de los problemas de las poblaciones de las que se hacen cargo (Castel, 2008). Focalizamos en algunas de las dinámicas desplegadas por los $\mathrm{CAB}$ que cumplen una función central en la asistencia dirigida a las expresiones más extremas de la exclusión social.

Sus técnicos han insistido en la singularidad de las situaciones e itinerarios de exclusión, sometiendo a las formas de vida asistidas a procesos de individualización que oscurecen los procesos de dominación, explotación y discriminación implicados en su destino. Tal como relata la joven profesional del $\mathrm{CAB}$ "cada usuario, al final tiene su propio recorrido, su propia historia, entonces cada uno marca sus objetivos" (CAB2). Por lo tanto, consideran que su labor debe centrarse sobre el individuo asistido, tratando de empoderarlo para que emprenda recorridos sociales de inserción:

No se trabaja en base a lo ajeno, sino a lo propio, porque también trabajar en base a lo que la sociedad ha podido afectar a esta persona no lo sé si tiene tanto sentido como trabajar en lo individual y en lo que cada uno también, puede tomar parte (CAB2).

Los profesionales asumen su incapacidad para transformar cuestiones estructurales: "lo social está ahí, sí, pero nosotros no lo podemos cambiar. Cada persona tiene que trabajar en base a lo suyo, a lo individual" (CAB2). Debido a su reducido margen de acción, los $\mathrm{CAB}$ despliegan políticas de la impotencia y el espectáculo (Fassin, 1999), mediante prácticas que tienen la función explícita de reducir el sufrimiento vital de los asistidos: talleres de distinto tipo (manualidades, lectura, relajación, fotografía entre muchas otras) y servicios básicos de higiene, lavandería y alimentación. Pero, más que mejorar las condiciones materiales de existencia de los asistidos en su camino hacia la integración, son prácticas cuyo cometido es el de mantenerlos ocupados.

Tal como afirma el director del CAB, "hay casos aquí que..., yo creo que podemos ver que no tratamos ni deberíamos tratar nunca de insertarlos en nada" (CAB3). Es decir, se asume que, tal como afirma Castel (1997), constituyen potencialmente un conjunto de seres humanos que son inintegrables. A pesar de la constatación de la imposibilidad de incorporar a determinados seres a las dinámicas sociales positivas, los técnicos continúan con un afán integrador explícito: "sería la de intentar ayudar a mejorar el proceso de inserción en su vida social, laboral y... económica ¿no?" (CAB4). Dicha vocación de devolver al rebaño a la oveja descarriada se realiza a través de una serie de técnicas de gobierno dirigidas a la reconstrucción del vínculo social.

En la intervención se considera que la integración pasa por la incorporación de un conjunto de normas y sentidos compartidos que articulan la vida colectiva (Ciapessoni, 2007). Hemos podido dilucidar cómo tres herramientas ocupan una posición 
central en esos procesos de reinserción: el calendario, el horario y la agenda. Por medio de estas herramientas, los regidores de los $\mathrm{CAB}$ tratan de incorporar en las dinámicas espacio-temporales de la vida social ordinaria a los usuarios, considerándolos tres ejes cardinales para la reconstrucción del lazo social de aquellos seres sumidos en los espacios negativos de lo social.

I) El calendario es una herramienta que posibilita que el asistido proyecte su propia planificación biográfica, tanto la trayectoria previa a estar en situación de calle, como la manera en la que se planea el futuro. En el terreno prima un discurso que plasma la imposibilidad de trazar una estrategia biográfica al margen de la integración, visibilizan una situación de stand-by, periodo de ruptura tan fuerte que les deja paralizados, como se señala en alguna ocasión. Se trata de un instrumento que posibilita la proyección hacia el futuro, la elaboración de un encadenamiento ordenado de las diferentes etapas por las que ha de discurrir la integración. En teoría, estas etapas actuarían como distintos hitos que poco a poco irán dando paso a la integración (en el caso de que no acontezca alguna otra ruptura):

Lo importante es que se sientan útiles, que su vida sea satisfactoria y que podamos trabajar sus potencialidades, punto. O sea, mucha gente va a terminar yendo a un centro de día toda su vida, y eso le obliga a madrugar, ducharse, vestirse e ir en autobús a no sé dónde y volver, y ya está. Ahí se relaciona... creo que la incorporación social es de muchas formas (CAB1).

El establecimiento de un calendario, permite la reubicación temporal de los individuos en la propia biografía, el ordenar los diferentes episodios biográficos que se esperan desarrollar. Desde algunas partes se mantiene el discurso de hacer que las personas "recuperen las riendas de su vida" (TEC1), y para que esto sea así, se introduce en ese orden, que pasa desde la búsqueda de trabajo, hasta la inscripción en diferentes cursos de formación o visitas al médico. La idea de ordenar las actividades (Ciapessoni, 2009) en un régimen de largo plazo demuestra la capacidad de planificación, la responsabilidad sobre el proyecto y el interés en construirlo. Parece mostrar un compromiso individual sobre ese futuro, de alguna manera, el incorporar esa preocupación se ve como el interés por la individualización de la responsabilidad, aunque por otro lado, se visualiza también la creencia de que ese individuo va a estar ya siempre ligado de alguna manera a la asistencia, de cuyas redes se hace dependiente.

¿Los procesos han salido bien?, bueno, pues en el momento de salida igual sí, pero ha tenido una recaída y vuelve a estar con nosotros... Bueno, ¿ha ido bien? Depende cómo midas... si tú mides que esa persona que ha estado bien y que ha tenido calidad de vida, pues entonces ha merecido la pena (...). El comedor no es sólo un sitio donde vienen a comer, es también un sitio de referencia para ellos (TEC2).

Hay un señor aquí que viene desde hace 20 años a comer (...), nos avisa cuando tiene citas médicas y ya está (...). Él viene nos cuenta su situación médica y ya está, pues bien, esa es su forma de relacionarse, pero también sabemos que si falta más de dos días seguidos algo pasa, entonces llamas al hospital, él ya sabe que nosotras estamos al tanto, a él eso le sirve, pues ya está. Esa es su red (CAB1). 
II) El horario actúa, discretamente, de una manera disciplinante, articulándose como uno de los elementos más estrictos del entramado asistencial — en especial, en comedores y albergues-. Una de las cuestiones que más veces mencionan los técnicos, además del orden para que todo funcione correctamente debido a la cantidad de personas, es el elemento de adecuación a los horarios, la responsabilidad sobre los mismos, como si constituyeran una manera de (re)aprender a estar en sociedad. En el caso del CAB en el que se realizó la observación participante, los horarios establecidos deben ser respetados y constituyen un elemento central en la organización de la cotidianeidad:

Un albergue es como la mili ¿no? Viven 40-50 personas y a las 09:00 tienes que salir y volver a las 20:00 de la noche. No tienes sitio para quedarte ahí, y estar ahí y estas cosas (USU1).

Si no has hecho la mili no sabes... Hay gente que viene aquí y... los horarios estrictos... hay gente que con los horarios no lo llevan bien y les parece muy eso, muy así... pero yo no. (...) Las normas no son nada... no me parecen nada... porque estamos aquí setenta, cada uno viene de donde viene... si no hay normas no se puede. Es como la mili. Yo lo comparo con la mili. Si no te ponen normas... va a explotar ahí, van a poner fuego (USU1).

La incorporación de los horarios representa la encarnación de las normas, por tanto también, la encarnación del propio proyecto de futuro, unida a la interiorización de un proyecto de sociedad determinado en donde el individuo se incorpora como una pieza más. Se nos hace ver la existencia de un tiempo no- disciplinado, no enfocado a nada en particular, "si hay algo que tiene esta gente es tiempo" (TEC2) nos decía una de las personas entrevistadas, y con esto, nos decía mucho más. Para los trabajadores del centro de día, es ese tiempo vacío el que lleva al consumo de substancias de manera autodestructiva además de a delinquir:

Entonces lo que le ha hecho estar en la calle es (...) no poder trabajar, y por el hecho de estar en la calle, probablemente esté con consumos porque la calle te va a llevar al consumo sí o sí, y que probablemente tengan antecedentes penales, porque terminarás delinquiendo si estás en la calle (CAB4).

Por ello, es necesario dotar a esas horas de contenidos, de funciones que reporten algo para la sociedad en la que se inserta, y por la propia socialización, reporta también al propio individuo que se siente participe de un todo común, el proyecto social. El horario como norma, guarda una relación muy directa con el modelo productivo, y una extensión de esa organización a los diferentes ámbitos de la vida, "no saben seguir un horario" (CAB1), se nos decía en otra ocasión, y ésta pasaba a ser la preocupación inicial en el proceso de integración, ya que iba a ser la pieza clave para la incorporación a un trabajo, o incluso para establecer vínculos sociales con otras personas que sí tienen incorporadas este ordenamiento. Esta inquietud muestra el afán de construcción del individuo moderno, pone en evidencia cómo éste sigue siendo el paradigma a aspirar, a pesar de que la sociedad que subyace no pueda ya vincularse enteramente con este ideal como muestra la creciente precarización en la que se ve inmerso el conjunto de la población. 
III) La agenda es una herramienta clave relacionada con el calendario y el horario. Se trata de proyectos unidos a la temporalidad, se dota de contenido a esas horas muertas, se busca una función al estar y por tanto al ser, una retroactividad con la sociedad para poder ser reconocido como miembro pleno de ella y no como icono de un afuera de la normalidad. Agenda también como imagen encarnada de temporalidades anteriores (consumo de drogas, problemas mentales, cárcel), temporalidades que dejaban su seña en lo más físico, el cuerpo, y en donde el proceso de reincorporación al sistema pasa indudablemente por una recuperación corporal. La nueva agenda ha de desterrar los hábitos y temporalidades anteriores:

En el centro de día con esa persona hacemos un plan de trabajo, adquiere unos compromisos y nosotros con él también. Entonces se compromete a venir al centro todos los días, se compromete a ser acompañado en la toma de decisiones, se compromete a tomar un tratamiento psiquiátrico $(\mathrm{CAB} 1)$.

Forma parte de ese proyecto de futuro, muestra de un compromiso y responsabilidad consigo mismo, podría hablarse incluso de un rito de paso hacia la re-incorporación. El trazado de unas metas que ir consiguiendo, sobre todo en las personas que han pasado por trayectorias biográficas más conflictivas, con consumo de drogas, la metáfora de la agenda ayuda a visibilizar la intención de no volver atrás. Una pretensión de avanzar, que se hace patente en los discursos de aquellos usuarios del centro de día que aparentemente se alejan de lo que definen como mundo negativo del consumo de drogas, para reincorporarse a la vida social normal.

En el centro de día sí que haríamos planes con ellos, seguimientos, acompañamientos. Acompañamiento no sólo físico, acompañamiento en la toma de decisiones. Lo hacemos mediante tutorías. Cada uno tenemos seis personas tutorizadas, y semanalmente yo me siento con cada una de estas personas el día estipulado, los miércoles a las 11:30. Él sabe que en ese momento nos juntamos, que tenemos una hora más o menos pactada, y entonces ya hemos hecho una 'evaluación de necesidades' (CAB1).

Restauración de un vínculo social que se pretende hacer visible por medio de la transformación del propio individuo, no hay lugar para un vínculo fuera de las normas, es el espacio donde se erige la vela y por tanto queda a oscuras, el secreto sagrado del que habla Durkheim (1982) en Las formas elementales de la vida religiosa sobre el que es condición de posibilidad toda sociedad: desarticular ese misterio, ese secreto, el espacio significador, conllevará la pérdida del estímulo atrayente del vínculo social. El vínculo se dispensará a través de las relaciones afectivas, por la autorrealización en la comunidad.

Calendario, horario y agenda se entienden como herramientas de apoyo en el proceso de integración de los seres expulsados de las dinámicas sociales instituidas. Ese instrumental parece ayudar a superar las incapacidades y rupturas que son atribuidas a la propia situación de sinhogarismo, de modo que se considera que sirven para capacitar a los excluidos a superar sus carencias. Son entendidos como elementos de apoyo para aquellos seres carenciados que en el discurso hegemónico "han llegado a ser considerados potencial e idealmente como agentes activos en la construcción de su propia existencia" (Rose, 1998: 39). Así, no es la sociedad quien debe modificar 
su funcionamiento para dejar de ser productora de residuos, sino que son éstos quienes deben transformarse, tomando conciencia de sus carencias y de su necesidad de mejorar, para reincorporarse a esas dinámicas sociales que previamente los habían expulsado. Este postulado se refleja en la lógica del acompañamiento, que impregna la red asistencial actual en la que frente a la imposición de criterios y objetivos unívocos producto del saber profesional, toma especial importancia las elaboraciones individuales del asistido. Tal como relata el director del centro:

Nosotros intentamos una deslocalización del saber, que el saber está del lado del sujeto (...). No se trata ni de que el saber esté; nosotros, de hecho, muchas veces nos hacemos diluir, desaparecer, que no se nos vea mucho (CAB3).

Evitando la imposición normativa, lo que se hace es, en palabras del joven de prácticas en el $\mathrm{CAB}$, "intentar ayudar a las demandas, sin obligar" (CAB4). Se trata de formas de gobierno que parecen invertir las dinámicas disciplinarias de la modernidad (Foucault, 2012), produciéndose una reversibilidad de las relaciones de autoridad: lo que inicialmente era una norma que debía imponerse, pasa a ser reformulada como una demanda por parte de los asistidos (Rose, 1998). Los asistidos pasan a ser definidos, no como el soporte pasivo de un conjunto de determinantes sociales, sino como "sujetos activos que participan de su propio gobierno" (Rose, 1999: 27).

Las formas de gobierno deben fomentar la toma de conciencia de su situación de dependencia, afrontarla mediante el autoperfeccionamiento para su reconstrucción como activos ciudadanos. En este sentido, parece que estas formas de gobierno forman parte de la metanarrativa del gerenciamiento que produce una imagen de nuestra situación como una cuestión de autoestima y empoderamiento (Rose, 1999). Son prácticas coherentes con el diagrama del actual régimen neoliberal, en el que la figura del emprendedor, agente responsable de sí mismo, se ha constituido como uno de los nuevos centros retóricos referenciales (ibídem).

\section{Discusión, conclusiones y futuras líneas de trabajo}

Aunque somos conscientes de la parcialidad de nuestras interpretaciones ante la irreductibilidad de la vida humana (Ferrándiz, 2011), hemos podido trasladar nuestra experiencia en un terreno concreto a escenarios más amplios, constatando la vigencia de análisis como los de Fassin $(1996 ; 1999 ; 2005)$, Rose $(1998 ; 1999)$ y Castel (2008).

Interesados en las formas de gobierno del contenido negativo de la vida colectiva, nos hemos focalizado en los $\mathrm{CAB}$, las tecnologías asistenciales centrales en el entramado asistencial dirigido a la gestión de la exclusión social extrema en la ciudad de Bilbao. Hemos podido atender a las dinámicas de esos espacios que actúan como tecnologías civilizadoras bajo la pulsión de integrar a los asistidos en la vida colectiva. En concreto, tres herramientas (calendario, horario y agenda) que definen la ubicación espacio-temporal del individuo ordinario, son definidos como mecanismos indispensables en el proceso de resocialización e incorporación del sujeto en la vida cotidiana; es decir, para la construcción de un ser humano normal, inserto en una temporalidad que establece orden en su día a día. Así se pretende dotar de nor- 
mas que sirvan tanto en su relación con la institución asistencial, como en su futura incorporación al resto de instituciones sociales.

En coherencia con las conceptualizaciones hegemónicas de la exclusión social, las dinámicas de los $\mathrm{CAB}$ están determinadas por discursos que no permiten atender a las causas estructurales implicadas en cada caso concreto, de modo que no suelen cuestionarse en profundidad las estructuras de poder que oprimen a los seres asistidos. En este sentido, mediante la lógica del acompañamiento parece ponerse en marcha un proceso de autorresponsabilización de los sujetos carenciados, obligados a tornarse en agentes activos de su bienestar. Por lo tanto, a pesar de asumir, en mayor o menor medida, la existencia de dinámicas que expulsan a los asistidos de la vida social instituida, las racionalidades y prácticas asistenciales desplegadas en los CAB focalizan sus esfuerzos en el empoderamiento individual de los usuarios para que emprendan su camino hacia la auto-mejora y su integración en la sociedad. En este sentido, parece plausible plantear la coherencia entre estas modalidades de gestión y la gubernamentalidad neoliberal. Por otro lado, hemos podido observar el modo en el que diversas formas de exclusión extrema conviven en los mismos recursos. Así consideramos necesario continuar nuestra labor investigadora para desvelar las diferentes experiencias que convergen bajo la categoría de PSH: no sólo abordando la distinción entre nuevos y viejos pobres, sino también aquellas situaciones determinadas por la condición de extranjero.

Ante el aumento de la precarización a todos los niveles, los estándares securitarios que alejaban al individuo-ciudadano de ser un posible empobrecido social han cambiado radicalmente. Derivado de ello, consideramos necesario atender al modo en el que se utilizan las redes asistenciales al tiempo que se observa cómo estas lógicas influyen en sus vivencias y configuran identidad en los individuos atravesados por los diferentes procesos de precarización contemporáneos.

\section{Bibliografía}

Agamben, G. (2015). ¿Qué es un dispositivo?. Barcelona: Anagrama.

Aierdi, X., Oleaga, J. A., Moreno, G., Herrero, I. y Alonso de Armiño, I. (2009). Perfil y necesidades de las Personas Sin Hogar en Bizkaia. Bilbao: Diputación Foral de Bizkaia.

Ararteko (2006). Respuesta a las necesidades básicas de las personas sin hogar y en exclusión grave. Bilbao: Ararteko.

Augé, M. y Colleyn, J. P. (2005). ¿Qué es la antropología?. Barcelona: Paidós Ibérica.

Autés, M. (2004). "Tres formas de desligadura". En S. Karsz (coord.), La exclusión: bordeando sus fronteras. Definiciones y matices (pp. 15-54). Barcelona: Gedisa.

Bauman, Z. (2005). Vidas desperdiciadas. La modernidad y sus parias. Madrid: Paidós.

Biehl, J. (2005a). VITA. Life in a Zone of Social Abandonment. Berkeley: University of California Press.

Biehl, J. (2005b). Technologies of Invisibility: Politics of Life and Social Inequality. En J. X. Inda (ed.), Anthropologies of Modernity. Foucault, Governmentality, and Life Politics (pp. 248-271). Oxford: Blackwell Publishing Ltd.

Biehl, J. (2007). A Life: Between Psychiatric Drugs and Social Abandonment. Subjectivity. Ethnographic Investigations, 397-421.

Bourdieu, P., Wacquant, L., Champagne, P. (1999). La miseria del mundo. Madrid: Akal. 
Bourgois, P. (2010). En busca de respeto. Vendiendo crack en Harlem. Buenos Aires: Siglo XXI.

Bourgois, P. y Schonberg, J. (2009). Righteous dopefiend. Berkeley: University of California Press.

Butler, J. (2002). Cuerpos que importan. Sobre los límites materiales y discursivos del sexo. Buenos Aires: Paidós.

Butler, J. (2010). Marcos de guerra. Las vidas lloradas. Barcelona: Paidós.

Cabrera, P. J. (1998). Huéspedes del aire: Sociología de las Personas Sin Hogar en Madrid. Madrid: Universidad Pontificia Comillas.

Cabrera, P. J. (2007). "Exclusión social: contextos para un concepto". Revista de Treball Social (TBS), 180, 9-21.

Campana, M. (2014). "Desarrollo humano, producción social de la pobreza y gobierno de la pobreza". Revista Trabajo Social, (16), 79-89.

Castel, R. (1997). La metamorfosis de la cuestión social: una crónica del salariado. Buenos Aires: Paidós.

Castel, R. (2008). La Inseguridad Social. ¿Qué es estar protegido?. Buenos Aires: Manantial.

Ciapessoni, F. (2007). "De refugios y calle: la construcción de la identidad en hombres sin domicilio". En M. De Martino y L. E. Morás (comps.), Sobre cercanías y distancias; problemáticas vinculadas a la fragmentación social en el Uruguay actual (pp. 103-120). Montevideo: Cruz del Sur.

Ciapessoni, F. (2009). Ajustes y desajustes: debates conceptuales sobre las poblaciones "sin domicilio". Montevideo: Proyecto CSIC.

Das, V., y Poole, D. (2008). "El Estado y sus márgenes. Etnografías comparadas". Revista Académica de Relaciones Internacionales, (8), 1-39.

De Marinis, P. (1999). “Gobierno, gubernamentalidad, Foucault y los anglofoucaultianos (o un ensayo sobre la racionalidad política del neoliberalismo)". In R. Ramos y F. García Selgas (comps.), Globalización, riesgo, reflexividad. Tres temas de la teoría social contemporánea (pp.). Madrid: Centro de Investigaciones Sociológicas.

Deleuze, G. (1990). “¿Qué es un dispositivo?”. En AA. VV., Michel Foucault, Filósofo (pp. 155-163). Barcelona: Gedisa.

Dubet, F. (2010). Sociología de la experiencia. Madrid: Complutense.

Durkheim, E. (1982). Las formas elementales de la vida religiosa. Madrid: Akal Editor.

Fassin, D. (1996). «Exclusion, Underclass, marginalidad: Figures contemporaines de la pauvreté urbaine en France ». Revue française de sociologie, 37 (1), 37-75.

Fassin, D. (1999). “La patetización del mundo: ensayo de antropología del sufrimiento". En M. Viveros y G. Garay (Eds.), Cuerpos, diferencias y desigualdades (pp. 31-41). Santafé de Bogotá: Utópica.

Fassin, D. (2005). "L'ordre moral du monde essai d'anthropologie de l'intolérable". En P. Bourdelais y D. Fassin, Les constructions de l'intolerable (pp.17-50). Paris: La Découverte.

Ferrándiz, F. (2011). Etnografías contemporáneas: anclajes, métodos y claves para el futuro. Madrid: Anthropos.

FOESSA (2014). VII Informe sobre exclusión y desarrollo en España. Madrid: Foessa.

FOESSA (2016). Expulsión social y recuperación económica. Madrid: Foessa.

Foucault, M. (1978). "La locura y la sociedad". En M. Foucault y M. Watanabe (Eds.), Tetsugaku no butai (pp. 63-76). Tokio: Asahi-Shuppansha.

Foucault, M. (2003). Hay que defender la sociedad. Madrid: Akal.

Foucault, M. (2008). Seguridad, territorio y población. Madrid: Akal. 
Foucault, M. (2012). Vigilar y castigar. Nacimiento de la prisión. Madrid: Biblioteca Nueva.

Gatti, G. (2014). "Y más allá de la identidad, la vulnerable víctima: zombis, llantos papales e inexistencia social". En I. Irazuzta y M. Martínez (Coords.), De la identidad a la vulnerabilidad. Alteridad e Integración en el País Vasco contemporáneo (pp. 195-209). Barcelona: Bellaterra.

Gordon, C. y Miller, P. (Eds.) (1991). The Foucault Effect: Studies in Governmentality. Chicago: The University of Chicago Press.

Gueslin, A. (1998). Gens pauvres, pauvres gens dans la France du xixe siècle. Paris: Aubier. Hacking, I. (1990). The Taming of Chance. Cambridge: Cambridge University Press

Hall, S. (2003). “¿Quién necesita identidad?”. En AA. VV., Cuestiones de identidad cultural (pp. 13-37). Barcelona: Amorrortu.

Herzog, B. (2011). "Exclusión discursiva. Hacia un nuevo concepto de la exclusión social". Revista Internacional de Sociología (RIS), 69(3), 607-626.

Inda, J. X. (Ed.) (2005). Anthropologies of Modernity. Foucault, Governmentality, and Life Politics. Oxford: Blackwell Publishing Ltd.

Irazusta, I. y Martínez, M. (2014). "De la identidad a la vulnerabilidad. La cuestión de la inmigración y las irrupciones en el nosotros”, en I. Irazusta \& M. Martínez, De la identidad a la vulnerabilidad. Alteridad e integración en el País Vasco contemporáneo (pp. 9-37). Barcelona: Ediciones Bellaterra.

Lewkowicz, I. (2004). Pensar sin Estado. La subjetividad en la era de la fluidez. Buenos Aires: Paidós.

Martín, A. (2011). "Diferentes prismas para estudiar la exclusión social. Marco teórico y propuesta de reconceptualización de la exclusión basado en el sujeto". Documentación de Trabajo Social, (49), 110-125.

Martuccelli, D. (2007). Gramáticas del individuo. Buenos Aires: Losada.

Miller, P. y Rose, N. (2008). Governing the Present. Administering Economic, Social and Personal Life. Cambridge: Polity Press.

Mussetta, P. (2009). "Foucault y los anglofoucaultianos: una reseña del Estado y la gubernamentalidad". Revista Mexicana de Ciencias Políticas y Sociales, 51(205), 37-55.

Narayan, D. (2002). La voz de los pobres: clamando por el cambio. Madrid: Mundi-Prensa Libros.

Paugam, S. (2007). Las formas elementales de la pobreza. Madrid: Alianza.

Pérez-Agote, A. (1996). "La sociedad se difumina, el individuo se disgrega. Sobre la necesidad de historizar nuestras categorías”, en A. Pérez Agote e I. Sánchez de la Yncera, (Ed.), Teoría social y complejidad (pp. 11-32). Madrid: CIS.

Raya, E. (2005). "Categorías sociales y personas en situación de exclusión. Una aproximación desde el País Vasco". Cuadernos de Relaciones Laborales, 23(2), 247-267.

Rose, N. (1998). "El gobierno en las democracias liberales avanzadas: del liberalismo al neoliberalismo". Cuadernos de critica de la cultura, (29), 25-40.

Rose, N. (1999). Governing the soul: the shaping of the private self. Londres: Free Association Books.

Silver, H. (1994). "Exclusión social y solidaridad social: Tres paradigmas". Revista Internacional del Trabajo, 113(5-6), 607-662.

Simmel, G. (2014). El pobre. Madrid: Sequitur.

Subirats, J. (2005). "Catorce puntos esenciales sobre evaluación de políticas públicas con especial referencia al caso de las políticas sociales". Ekonomiaz, 1(60), 18-37.

Tejerina, B., Cavia, B., Santamaría, E., y Carbajo, D. (2011). Precariedad vital y juventud 
vasca. Condiciones sociales y estrategias biográficas para llevar una vida normal. Bilbao: Observatorio Vasco de la Juventud.

Tocqueville, A. (2003). Memoria sobre el pauperismo. Madrid: Tecnos.

Velasco, H. y Díaz de Rada, Á. (2004). La lógica de la investigación etnográfica. Un modelo de trabajo para etnógrafos de la escuela. Madrid: Trotta.

Wacquant, L. (2001). Parias urbanos: marginalidad en la ciudad. Buenos Aires: Manantial. Wacquant, L. (2012). Merodeando las calles. Trampas de la etnografia urbana. Barcelona: Gedisa.

Willis, P. (2000). The Ethnographic Imagination. Cambridge: Polity Press. 